\title{
Vibration Isolation System with Kalman Filter Estimated Acceleration Feedback: An Approach of Negative Stiffness Control
}

\author{
Mhia Md Zaglul Shahadat $\left(\mathbb{D},{ }^{1}\right.$ Takeshi Mizuno, ${ }^{2}$ Masaya Takasaki, ${ }^{2}$ Fazlur Rashid $\left(\mathbb{D},{ }^{1}\right.$ \\ and Yuji Ishino ${ }^{2}$ \\ ${ }^{1}$ Department of Mechanical Engineering, Rajshahi University of Engineering \& Technology, Rajshahi-6204, Bangladesh \\ ${ }^{2}$ Department of Mechanical Engineering, Saitama University, Shimo Okubo 255, Sakura-Ku, Saitama 338-8570, Japan \\ Correspondence should be addressed to Mhia Md Zaglul Shahadat; mhiashahadat@gmail.com \\ and Fazlur Rashid; frrashed10@gmail.com
}

Received 19 June 2021; Accepted 12 November 2021; Published 16 December 2021

Academic Editor: Christos Riziotis

Copyright (c) 2021 Mhia Md Zaglul Shahadat et al. This is an open access article distributed under the Creative Commons Attribution License, which permits unrestricted use, distribution, and reproduction in any medium, provided the original work is properly cited.

\begin{abstract}
This paper presents the isolation of vibration through the acceleration feedback of the Kalman filter. In this paper, vibration isolation was analyzed both analytically and experimentally through the estimation of the Kalman filter (KF). A negative stiffness mechanism was used to reduce the level of vibration for the developed dynamic system. The technique of negative stiffness can provide stiffness of infinite level to low stiffness as well as disturbance generated by the ground vibration directly. The performance of an isolation system through a mechanism of negative stiffness was improved by the addition of acceleration feedback. Acceleration was measured using a microelectromechanical (MEMS) type accelerometer instead of traditional servo type accelerometers due to lower cost. However, the output of a microelectromechanical (MEMS) type accelerometer is usually noisy. To avoid this difficulty, an acceleration that was estimated by a Kalman filter was considered in the acceleration feedback instead of directly measured acceleration. The dynamic behaviors of the system were compared for both the Kalman-filtered acceleration and the directly measured acceleration feedback. It is observed that the former has a significant effect on the improvement of the characteristics of the vibration isolation systems than later.
\end{abstract}

\section{Introduction}

Vibrations are needed to be isolated in all different types of mechanical systems including high-performance Nano and microscale production processes. It is expected that mechanical systems work without any surrounding disturbances. To meet this demand, isolation systems such as microvibration are being heavily used in space equipment, semiconductor, and silicon wafer industries. In practical applications, vibration sources are considered as either ground vibration or onboard-generated direct disturbance [1-3]. An isolation system developed for vibration is able to suppress and reduce these disturbances simultaneously. A lower level of stiffness systems is appropriate for the attenuation of ground vibration whereas high-stiffness systems are appropriate for direct disturbances [4-6]. Moreover, a compensation of the performances of low- and high-stiffness systems is signifi- cant in the traditional passive vibration control; therefore, passive type systems for vibration isolation are limited in applications [7-9]. On the other hand, the principle of an active isolation system for vibration is safe with respect to this type of difficulty. Recently, the use of active microvibration isolation technology to isolate disturbances has received adequate attention in Hi-tech industries [10-13].

Previously, several approaches were used for realizing active vibration isolation systems. Among these methods, the series combination of two isolators is an effective method of maintaining simultaneous high-stiffness and low-stiffness suspension [14]. Meanwhile, various control strategies, such as state feedback control [15], $\mathrm{H}^{\infty}$ control [16], feedforward control [17], repetitive [18], and active acceleration control [19], were previously applied to achieve an active isolation system for vibration. In this work, a horizontal type isolation mechanism for vibration was developed based on the 
concept of negative stiffness technique where isolators of equal magnitude for positive as well as negative stiffness are connected in series connection.

A suspension of magnetic dynamic system with the control of zero-power possesses negative type stiffness. The negative stiffness of a zero-power control system is constant, and it varies depending on the constants of the magnet such as coefficients of permanent type magnet used to supply bias type force in the steady state, whereas an actuator (linear) with exact negative stiffness control gives a variable negative stiffness according to the gain selected [20]. In this current research work, the positive, as well as negative stiffness's, is realized by proportional control and proper negative stiffness control, respectively.

Nevertheless, the transient displacements of the vibration isolation systems mentioned above are not satisfactory in some cases and are needed to be suppressed. In a previous study, Hoque et al. improved the transient characteristics of the isolation of a vibration system by adding a feedforward control [21]. In this research, an innovative approach that comprises feedback of acceleration added to the isolation system for vibration attained with the negative stiffness mechanism. This approach could improve the characteristics of vibration isolation independently of the disturbances identified in the system $[21,22]$. MEMS accelerometers, which are low in cost compared to commercial servo accelerometers, are used to find acceleration. However, MEMS accelerometers have less sensitivity in a low-frequency domain compared to the servo accelerometers [23]. In principle, the zero-compliance system based on series connected isolators can maintain its position at lower level of frequency; hence, the nature of the developed dynamic system for the isolation of vibration at low frequency is not hampered with MEMS accelerometers. Nevertheless, the MEMS accelerometer carries undesirable noise which may cause the deterioration in dynamic responses [24]. Previously, various filtering techniques are used to improve the feedback signals as well as to reduce the effect of undesired noises $[25,26]$. To avoid the difficulty regarding noises carried by the MEMS accelerometers, this work uses the Kalman filter (KF) to estimate the acceleration using direct measured acceleration and system inputs; this estimated signal is supposed to be used for the acceleration feedback. A previous study showed that the Kalman filter performs suitably to improve the accuracy of the rotor position in active magnetic bearings [27].

\section{Negative Stiffness Mechanism for Vibration Isolation}

There are two conditions required to isolate a system from vibration, such as lower level of stiffness suspension to ground vibration and stiffness of infinite level against direct disturbance. However, these two criteria need to be activated simultaneously. In this work, the mechanism of negative stiffness is utilized to obtain these two required criteria simultaneously where two types of isolators are connected in series (Figure 1).
In the mechanism of negative stiffness, one isolator is designed that has a stiffness of positive magnitude and the other isolator provides stiffness with negative values where the magnitudes of stiffness were the same. In this work, the object was displaced in the opposite direction of the applied force. If $\delta l$ is the amount of movement in the upward direction due to the application of mass $\Delta m$ (Figure 1(a)), then negative stiffness is usually presented using the following equation.

$$
k=\frac{\Delta m g}{l-(l+\delta l)}=\frac{(\Delta m g)}{-\delta l}
$$

where the distance between the ground (base) and the object at steady state is denoted by 1 .

In contrast, in a positive stiffness mechanism, a system displaces in the exact same direction of force. Any weight with a system of spring-damper usually compresses when applying compression loads, while expands for tensile loads. If $k_{s}$ and $k_{p}$ are the coefficients of two isolators that are attached in series connection (Figure 1(b)), the combined stiffness " $k_{c}$ " can be obtained by the following Equation (2):

$$
k_{c}=\frac{k_{p} k_{s}}{k_{p}+k_{s}} .
$$

It is seen that if the stiffness of two individual isolators is positive (traditional spring) then the magnitude of combined stiffness $k_{c}$ is lower when compared to their individual stiffness. However, if one isolator has a positive stiffness and another one possesses a negative stiffness with equal in magnitude but opposite in direction $\left(\left|k_{p}\right|=\left|-k_{s}\right|\right)$, then their combined stiffness $k_{c}$ is infinite that can be presented by the following equation.

$$
k_{c}=\frac{\left(-k_{s}\right) k_{s}}{-k_{s}+k_{s}}=\infty
$$

Hence, in Equation (3), the relative distances of the top isolation table are zero with respect to the ground (base) against a force. In this research work, both isolators of positive as well as negative stiffness are realized by applying exact negative stiffness and proportional derivative controls.

\section{Modeling and Controller Design}

The designed experimental isolation system for vibration is a two-stage mechanism where the isolation and mass of the middle table are added in series connection. A dynamic model consists of a voice coil motor as actuators as depicted in Figure 2(a). Acceleration feedback was used to improve the dynamics of the developed negative stiffness-controlled system for the isolation of vibration. Importantly, this approach is independent of the disturbances identified in the system.

MEMS accelerometer was used to measure absolute acceleration that is used as acceleration feedback in each controller to identify the effect of the feedback of acceleration 


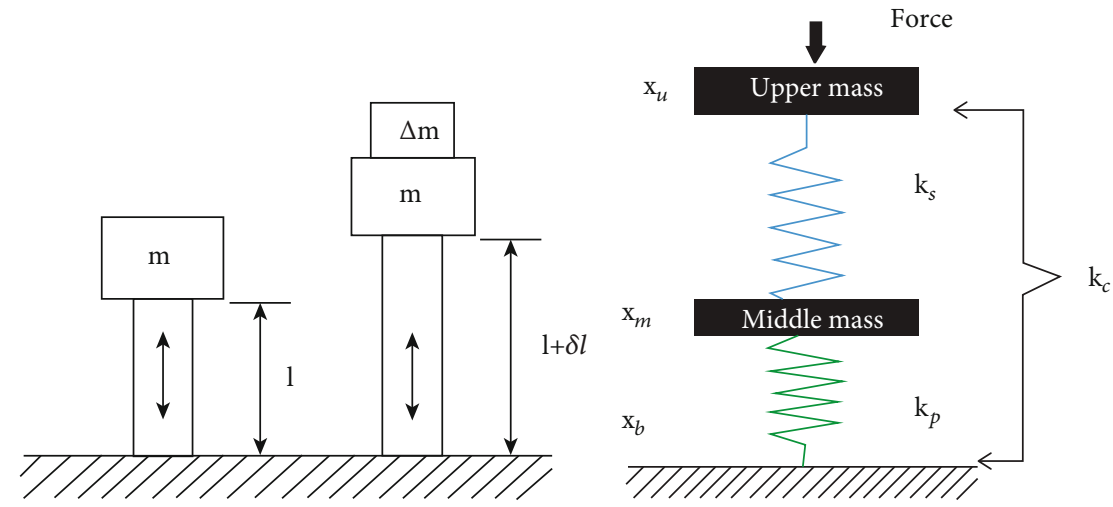

(a)

(b)

FIGURE 1: (a) Conceptual schematic of negative stiffness method. (b) Isolators in series connection.

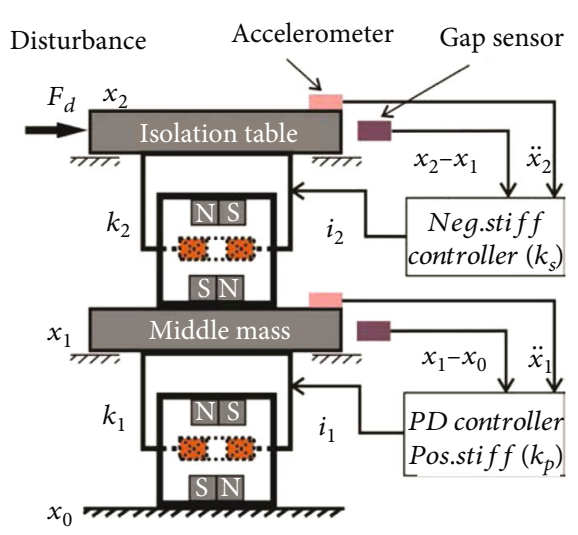

(a)

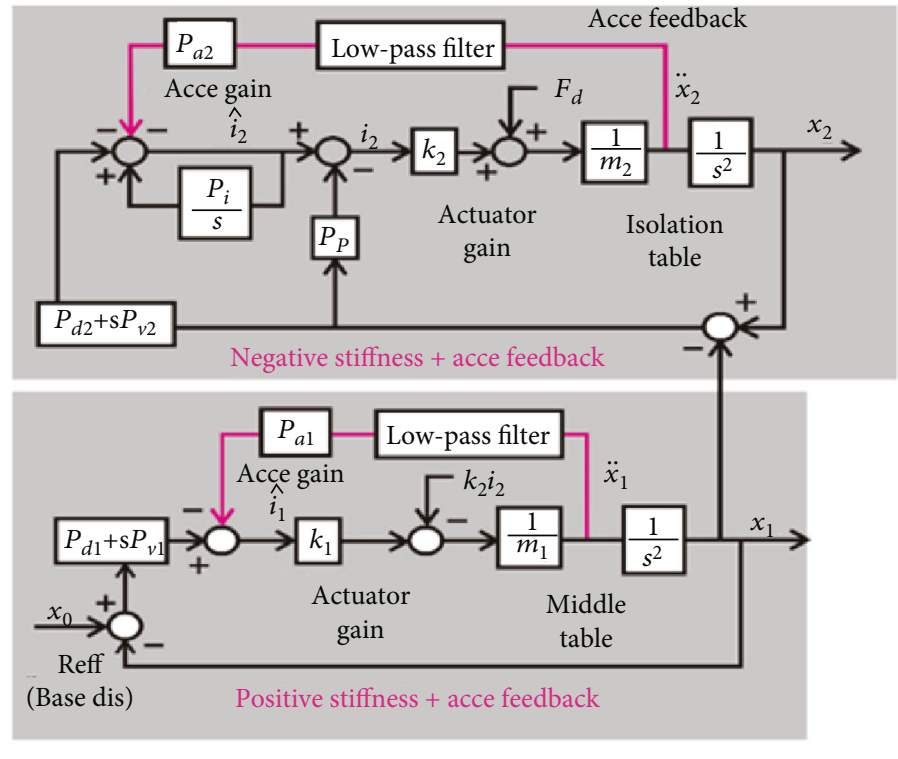

(b)

FIGURE 2: (a) Schematic diagram of the developed experimental setup with controller and (b) controllers along with acceleration feedback.

on the isolation characteristics of vibration. The table for vibration isolation (upper table in Figure 2(a)) is moved along the horizontal translation motion ( $x$-axis) where there is no interference (internal) from the remaining axes. The isolation motion for vibration and mas of the middle table can be written using these two equations:

$$
\begin{aligned}
& m_{1} \ddot{x}_{1}=k_{1} i_{1}-k_{2} i_{2}, \\
& m_{2} \ddot{x}_{2}=k_{2} i_{2}+F_{d},
\end{aligned}
$$

where $m$ denotes the table mass, $x$ denotes distances/displacements, $k$ is actuator thrust coefficient, and $F_{d}$ is the amount of direct disturbance. In addition, the subscript 0 presents the base, 1 denotes the middle table, and 2 presents the table for vibration isolation.
By transforming the equations using the Laplace transform, it is possible to obtain the following two Equations (5) and (6):

$$
\begin{aligned}
& m_{1} s^{2} X_{1}(s)=k_{1} I_{1}(s)-k_{i 2} I_{2}(s), \\
& m_{2} s^{2} X_{2}(s)=k_{2} I_{2}(s)+F_{d}(s) .
\end{aligned}
$$

The controller block diagram that is related to the mechanism of negative stiffness involves additional feedback of acceleration (Figure 2(b)). The middle mass was subjected to the proportional derivative controller, while in the isolation table, negative stiffness control was applied. Additional feedback of acceleration was added in both the two controllers. Hence, the currents for proportional-derivative (PD) 
control and negative stiffness based on the model can be written as follows:

$$
\begin{gathered}
I_{1}(s)=-\left(P_{d 1}+s P_{v 1}\right)\left(X_{1}-X_{0}\right)-s^{2} G_{F}(s) P_{a 1} X_{1}, \\
I_{2}(s)=\frac{-s^{3} G_{F} P_{a 2} X_{2}-\left(s^{2} P_{v 2}+s\left(P_{d 2}+P_{p}\right)-P_{p} P_{i}\right)\left(X_{2}-X_{1}\right)}{\left(s-P_{i}\right)},
\end{gathered}
$$

where $P_{d}$ is proportional, $P_{v}$ is derivative, $P_{i}$ is integral, $P_{p}$ is adjustment of stiffness, and $P_{a}$ is gains of acceleration feedback. On the other hand, $G_{F}$ presents the transfer function of a low-pass filter that is used to decrease the noisy signal presents in the feedback of acceleration.

In the case of isolation table for vibration, the transfer function of the ratio of distance $\left(x_{2}\right)$ to direct disturbance $\left(F_{d}\right)$ and distance $\left(x_{2}\right)$ to vibration generated by ground $\left(x_{0}\right)$ can be generated using the Equations (5) and (6) as well as (7) and (8).

$$
\begin{gathered}
\frac{X_{2}(s)}{F_{d}(s)}=\frac{\alpha_{3} s^{3}+\alpha_{2} s^{2}+\alpha_{1} s+\alpha_{0}}{\gamma_{5} s^{5}+\gamma_{4} s^{4}+\gamma_{3} s^{3}+\gamma_{2} s^{2}+\gamma_{1} s+\gamma_{0}}, \\
\frac{X_{2}(s)}{X_{0}(s)}=\frac{\beta_{3} s^{3}+\beta_{2} s^{2}+\beta_{1} s+\beta_{0}}{\gamma_{5} s^{5}+\gamma_{4} s^{4}+\gamma_{3} s^{3}+\gamma_{2} s^{2}+\gamma_{1} s+\gamma_{0}},
\end{gathered}
$$

where $\alpha_{3}=\left(m_{1}-k_{1} G_{F} P_{a 1}\right), \alpha_{2}=\left(-m_{1} P_{i}+k_{1} P_{v 1}+k_{1} G_{F} P_{a 1}\right.$ $\left.P_{i}+k_{2} P_{v 2}\right), \alpha_{1}=\left(k_{1} P_{d 1}-k_{1} P_{v 1} P_{i}+k_{2} P_{d 2}+k_{2} P_{p}\right), \alpha_{0}=\left(-k_{1}\right.$ $\left.P_{d 1} P_{i}-k_{2} P_{p} P_{i}\right), \beta_{3}=k_{1} k_{2} P_{v 1} P_{v 2}, \beta_{2}=k_{1} k_{2}\left(P_{d 1} P_{v 2}+P_{d 2} P_{v 1}+\right.$ $\left.P_{p} P_{v 1}\right), \beta_{1}=k_{1} k_{2}\left(P_{d 1} P_{d 2}+P_{d 1} P_{p}-P_{p} P_{i} P_{v 1}\right), \beta_{0}=k_{1} k_{2}\left(-P_{d 1}\right.$ $\left.P_{p} P_{i}\right), \gamma_{5}=\left(m_{1} m_{2}-m_{1} k_{2} G_{F} P_{a 2}-m_{2} k_{1} G_{F} P_{a 1}\right), \gamma_{4}=\left\{-m_{1} m_{2} P_{i}+\right.$ $\left.m_{2}\left(k_{1} P_{v 1}+k_{1} G_{F} P_{a 1} P_{i}+k_{2} P_{v 2}\right)+m_{1} k_{2} P_{v 2}-k_{1} k_{2} G_{F} P_{a 1} P_{v 2}\right\}$, $\gamma_{3}=\left\{\left(m_{1}+m_{2}\right)\left(k_{2} P_{d 2}\right)+\left(m_{2}-m_{1}\right)\left(k_{2} P_{p}\right)+m_{2}\left(k_{1} P_{d 1}-k_{1} P_{v 1} P_{i}\right)+\right.$ $\left.k_{1} k_{2}\left(P_{a 1} P_{d 2}+P_{a 1} P_{p}+P_{v 1} P_{v 2}\right)\right\}, \gamma_{2}=\left\{\left(m_{1}-m_{2}\right)\left(k_{2} P_{p} P_{i}\right)+\right.$ $\left.m_{2}\left(-k_{1} P_{d 1} P_{i}\right)+k_{1} k_{2}\left(P_{d 2} P_{v 1}+P_{p} P_{v 1}+G_{F} P_{a 1} P_{p} P_{i}+P_{d 1} P_{v 2}\right)\right\}$, $\gamma_{1}=\left\{k_{1} k_{2}\left(-P_{p} P_{v 1} P_{i}+P_{d 1} P_{d 2}+P_{d 1} P_{p}\right)\right\}, \gamma_{0}=k_{1} k_{2}\left(-P_{d 1} P_{p} P_{i}\right)$.

The acceleration feedback gains $P_{a 1}$ and $P_{a 2}$ determine the coefficient $s^{5}\left(\gamma_{5}\right)$ as a rise of these gains negatively causes the reduction of the numerator of Equations (9) and (10). Theoretically, it is seen that additional feedback of acceleration reduces the displacements of a dynamic system when compared to no acceleration feedback condition.

The rise of the gains of feedback of acceleration in the controller causes a mass increment of a dynamic system (coefficient of $s^{5}\left(\gamma_{5}\right)$ ). Therefore, in practice, the system would be stable in certain ranges of $P_{a 1}$ and $P_{a 2}$. The gains of acceleration are chosen in such a way to the system stable. However, to find the displacement of the tables at steady state in the form of disturbance generated directly, $F_{d}$ is taken as stepwise, while the ground vibration is zero $\left(x_{0}=0\right)$. It can be determined by using the following equations:

$$
\frac{X_{2}(\infty)}{F_{0}}=\lim _{s \longrightarrow 0} \frac{\alpha_{3} s^{3}+\alpha_{2} s^{2}+\alpha_{1} s+\alpha_{0}}{\gamma_{5} s^{5}+\gamma_{4} s^{4}+\gamma_{3} s^{3}+\gamma_{2} s^{2}+\gamma_{1} s+\gamma_{0}}=\frac{\alpha_{0}}{\gamma_{0}},
$$

$$
\frac{X_{2}(\infty)}{F_{0}}=\frac{-k_{1} P_{d 1} P_{i}-k_{2} P_{p} P_{i}}{k_{1} k_{2}\left(-P_{d 1} P_{p} P_{i}\right)}=\frac{-1}{k_{2} P_{p}}+\frac{1}{k_{1} P_{d 1}}=-\frac{1}{k_{s}}+\frac{1}{k_{p}} .
$$

The negative and positive stiffness of the given system can be determined through Equation (12). It is seen that if the magnitudes (absolute) of positive as well as negative stiffness are zero then the steady-state type displacement of the developed table for vibration isolation is zero. Therefore, a system with negative stiffness as well as additional acceleration feedback can be used to improve the isolation behavior of vibration.

3.1. Controller Parameters. In this work, the magnitudes of gains of controller parameters are determined using the method of pole assignment. However, the developed vibration control system with negative stiffness mechanism approaches a fifth order of system where the determination of acceleration feedback gains using pole assignment is complex and tedious.

To avoid such complexity, the middle and isolation table is assumed to be the individual systems where the positive and negative stiffness controls are applied, respectively. A basic one degree-of-freedom (DOF) model is considered to determine controller gains (Figure 3). The motion of the table can be determined by the following equation:

$$
m \ddot{x}=F_{a}+F_{d},
$$

where $m$ is table mass, $x$ is distance of the table along the $x$ -axis, thrust force of actuator is $F_{a}$, and $F_{d}$ is the direct disturbance of the table.

The force $F_{a}$ can be calculated by the following equation.

$$
F_{a}=k_{i} i
$$

where generated thrust of the voice coil motor (VCM) is proportional to the coil current $i$ and $k$ is the thrust coefficient.

The transfer function of the actuator dynamics can be written using the Laplace transforms by the following equation:

$$
X(s)=\frac{1}{s^{2}}\left(\frac{k_{i}}{m} I(s)+\frac{1}{m} F_{d}(s)\right)
$$

where the variable of each Laplace transform is denoted by their capital.

3.1.1. Negative Stiffness Control. The control mechanism using negative stiffness consists of a proportional-derivative (PD) and local integral feedback (Figure 2(b)). The current 


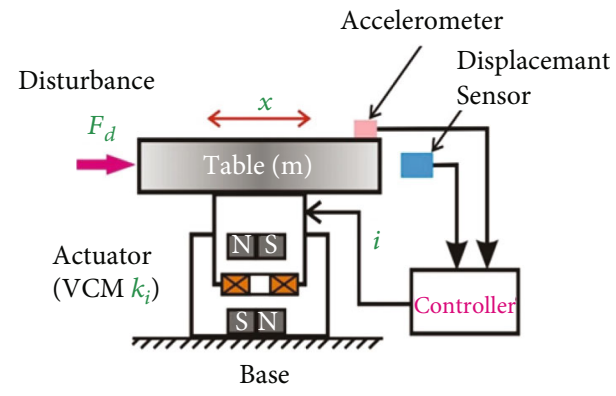

Figure 3: Single-axis horizontal suspension system.

( $i$, Figure 3) for the negative stiffness control can be written using the following equation of Laplace transform.

$$
\left.I(s)=\left(-\frac{P_{d 2}+s P_{v 2}}{1-P_{i} / s}+P_{p}\right)\left(X(s)-X_{0}(s)\right)-\frac{s^{2} P_{a 2}}{1-P_{i} / s} X(s)\right),
$$

where $P_{d 2}$ is proportional, $P_{v 2}$ is derivative, $P_{i}$ is integral, $P_{p}$ is stiffness adjustment, and $P_{a 2}$ is acceleration feedback gains.

Finally, the representation of the transfer function of the dynamics of the system with respect to the displacement to the vibration generated by ground and direct disturbance can be determined by the following two equations.

$$
\begin{gathered}
\frac{X(s)}{F_{d}(s)}=\frac{\left(s-P_{i}\right)}{t_{c}(s)\left(m+k_{i} P_{a 2}\right)}, \\
\frac{X(s)}{X_{0}(s)}=\frac{\left(k_{i} P_{v 2}\right) s+\left(k_{i} P_{d 2}-k_{i} P_{p}\right) s+k_{i} P_{p} P_{i}}{\left(m+k_{i} P_{a 2}\right) \cdot t_{c}(s)},
\end{gathered}
$$

where $t_{c}(s)$ indicates the characteristics equation of the system (Figure 3) controlled with negative stiffness control, which is extended by acceleration feedback and is expressed as follows:

$t_{c}(s)=s^{3}+\left(\frac{k_{i} P_{v 2}-m P_{i}}{m+k_{i} P_{a 2}}\right) s^{2}+\left(\frac{k_{i} P_{d 2}-k_{i} P_{p}}{m+k_{i} P_{a 2}}\right) s+\frac{k_{i} P_{p} P_{i}}{m+k_{i} P_{a 2}}$.

Equation (19) shows that a dynamic system with the control of negative stiffness behaves as a third-order system. In general, the characteristic ideal equation of a third-order system can be expressed by using the following equation.

$$
t_{d}(s)=\left(s^{2}+2 \zeta_{1} \omega_{1} s+\omega_{1}^{2}\right)\left(s+\omega_{2}\right)=s^{3}+\lambda_{2} s^{2}+\lambda_{1} s+\lambda_{0},
$$

where $\lambda_{2}=2 \zeta \omega_{1}+\omega_{2}, \lambda_{1}=2 \zeta \omega_{1} \omega_{2}+\omega_{1}^{2}$, and $\lambda_{0}=\omega_{1}^{2} \omega_{2}$. In determining the gains, it is assumed that $\omega_{1}=\omega_{2}=\omega_{r}$. The symbols $\omega_{r}\left(2 \pi f_{r}\right)$ present the frequency (angular), and $\zeta_{r}$ denotes the damping ratio. In general, these two variables can be used to characterize the dynamics of a second-order system. Here, these parameters are used to find the poles of the closed-loop of the third-order system, as they are comprehensive when compared to the poles themselves.

Finally, the controller gains are calculated by the comparison of Equations (19) and (20) that can be written by the following equation.

$$
\begin{aligned}
P_{p} & =\frac{k_{s}}{k_{i}}, P_{i}=\frac{\alpha_{0}}{k_{i} P_{p}}\left(m+k_{i} P_{a 2}\right), P_{d 2} \\
& =\frac{1}{k_{i}}\left(\alpha_{1}\left(m+k_{i} P_{a 2}\right)+k_{i} P_{p}\right), P_{v 2} \\
& =\frac{1}{k_{i}}\left(\alpha_{2}\left(m+k_{i} P_{a 2}\right)+m P_{i}\right) .
\end{aligned}
$$

3.1.2. Positive Stiffness Control (PD Controller). Likewise, the gains for the proportional-derivative controller (positive stiffness) are determined with respect to the same model. A diagram of the proportional-derivative control, including the feedback of acceleration, is depicted in Figure 2(b). The representation of the transfer function of the table (displacement to disturbance measured directly and vibration from base/ground) with proportional derivative control can be written using these two following equations.

$$
\begin{gathered}
\frac{X(s)}{F_{d}(s)}=\frac{1}{\left(m+k_{i} P_{a 1}\right) \widehat{t}_{c}(s)}, \\
\frac{X(s)}{X_{0}(s)}=\frac{\left(k_{i} P_{v 1}\right) s+k_{i} P_{d 1}}{\left(m+k_{i} P_{a 1}\right) \widehat{t}_{c}(s)},
\end{gathered}
$$

where $\hat{t}_{c}(s)$ presents the characteristic equation of the dynamic system as written by the following equation.

$$
\widehat{t}_{c}(s)=s^{2}+\left(\frac{k_{i} P_{v 1}}{m+k_{i} P_{a 1}}\right) s+\frac{k_{i} P_{d 1}}{m+k_{i} P_{a 1}},
$$

where $P_{d 1}$ is proportional, $P_{v 1}$ is derivative, and $P_{a 1}$ is acceleration feedback gains of the proportional-derivative (PD) controller. It is because systems with a traditional PD control are second-order systems where the gains for the developed control system are obtained by the comparison of the coefficients of the equation of an ideal secondorder system with Equation (24). In general, the equation of an ideal system of second order can be written by the following equation.

$$
\widehat{t}_{d}(s)=\left(s^{2}+2 \widehat{\zeta}_{r} \widehat{\omega}_{r} s+\omega \wedge_{r}^{2}\right)=s^{2}+\rho_{1} s+\rho_{0},
$$

where angular frequency and ratio of damping use to find the poles of the second-order closed-loop system. Finally, the controller gains for the PD controller are calculated by using the following equation.

$$
\begin{aligned}
& P_{d 1}=\frac{m \rho_{0}}{k_{i}}, \\
& P_{v 1}=\frac{m \rho_{1}}{k_{i}} .
\end{aligned}
$$


Moreover, the proportional gain $\left(P_{d 1}\right)$ of the controller shows the static positive stiffness of the controlled system. Hence, the proportional gain $\left(P_{d 1}\right)$ is calculated in such a way that the acquired positive type stiffness $\left(k_{p}\right)$ and the absolute magnitude of the negative type stiffness $k_{s}$ are equal. Hence, the proportional gain $\left(P_{d 1}\right)$ can be determined through the following equation.

$$
P_{d 1}=\frac{k_{p}}{k_{i}} .
$$

In the theoretical analysis of designing the controllers (mentioned above), it is found that the consideration of feedback of acceleration with controllers virtually increases the mass of the system, which leads to a smaller displacement against disturbance (known or unknown). Moreover, it is identified theoretically from Equations (17) and (22) that the dynamic displacement will decrease as the acceleration gains $\left(P_{a 1}\right.$ and $\left.P_{a 2}\right)$ increase. However, in practice, the system would be unstable beyond a certain range of $P_{a 1}$ and $P_{a 2}$; hence, the values of $P_{a 1}$ and $P_{a 2}$ are adjusted so that the system stands within stable region.

3.2. Optimal Estimation Using Kalman Filter. An accelerometer is usually used to find the absolute value of the acceleration of a system. However, a commercial accelerometer of servo type is usually expensive. To solve this issue, a microelectromechanical system (MEMS) accelerometer is utilized that is cheap but the signals are noisy. Also, the MEMS accelerometer requires less space that could increase the overall dimension of the setup.

To reduce such noise, the Kalman filter estimated values were used instead of directly measured values for the feedback signal. The measurement of the Kalman filter can be considered as an algorithm of linear quadratic estimation (LQE) that can be determined by the measurement of a series of data (noise and other inaccuracies) over time.

These estimates of variables are more precise when compared to a single measurement. The significant application of the Kalman filter is to use in the navigation and control of aircraft, spacecraft, etc. [28]. The Kalman filter used in this work is an observer-based filter (Figure 4), which estimates the value of acceleration by reducing the estimation of the errors of mean square (MSE). As a consequence, the efficiency of the vibration isolation system will increase.

3.2.1. Simulations. The state-space model of the system including process as well as measurement noise can be shown by the following two equations.

$$
\begin{aligned}
& \dot{x}=A x+B u+q, \\
& y=C x+r,
\end{aligned}
$$

where $x$ denotes state variables, $A$ presents state transition, $B$ shows control input, $C$ is measurement matrices, $u$ is input variable vector, $q$ denotes process vector for the estimation of noise, and $r$ is measurement noise vector. The

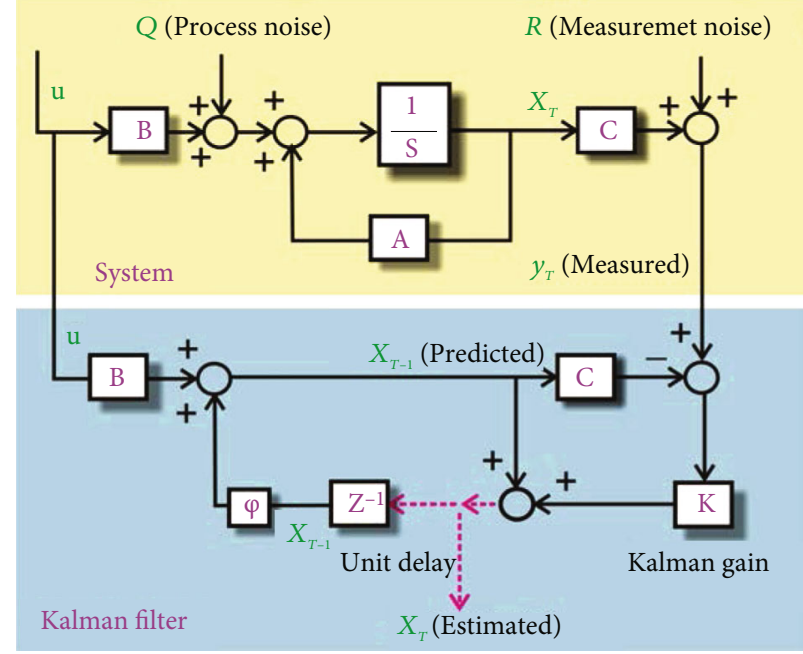

FIgURE 4: Block diagram of the Kalman filter (KF) algorithm.

discrete model of state-space for the dynamic system can be written using the following two equations with sampling time.

$$
\begin{aligned}
& x_{k}=\Psi x_{k-1}+\Phi u+\gamma, \\
& y_{k}=C x_{k}+\lambda y=C x+r,
\end{aligned}
$$

where $\Psi=A \Delta t+I, \Phi=B \Delta t, \gamma=q \Delta t, \lambda=r \Delta t$, and $k$ presents the time step. The estimation of the Kalman filter values consists of two processes (state prediction and state correction) individually, and the outcome of each step is utilized as input to another step and continues these until the iteration of the last value. To find these two steps/processes, the algorithm of the Kalman filter is performed in two different processes such as the time as well as measurement update.

(1) Time Update.

$$
\begin{aligned}
x_{k-1} & =\Psi \widehat{x}_{k-1}+B u, \\
P_{k-1} & =\Psi \widehat{P}_{k-1} \Psi^{\prime}+Q .
\end{aligned}
$$

(2) Measurement Update.

$$
\begin{aligned}
& \widehat{x}_{k}=x_{k-1}+K_{k}\left(y_{k}-C x_{k-1}\right), \\
& K_{k}=\left(P_{k-1} C^{\prime}\right)\left(C P_{k-1} C^{\prime}+R\right)^{-1}, \\
& \widehat{P}_{k}=\left(I-K_{k} C\right) \widehat{P}_{k-1},
\end{aligned}
$$

where $Q$ denotes covariance matrices of noise (process), $R$ shows noise due to measurement, $P$ is estimation error, and $K_{k}$ is Kalman gain matrix. The predicted and the estimated value of $x$ is denoted by $(x)$ and $(\widehat{x})$. In this work, several numerical simulations were performed to identify the influence of the feedback of acceleration based on the estimation of Kalman filter values on the isolation of vibration. The input, state variable and transition, and matrices 
TABLE 1: Parameters used in the experiments and simulations.

\begin{tabular}{|c|c|c|c|c|}
\hline Model & Parameters & & Value & Unit \\
\hline \multirow{6}{*}{ Kalman filter } & \multirow{3}{*}{ Process noise $(Q)$} & \multirow{3}{*}{$0.02 I_{5 \times 1}$} & \multirow[t]{2}{*}{0.02 eye (3) negative stiff system } & $\begin{array}{l}\mathrm{m} / \mathrm{s} \\
\mathrm{m} / \mathrm{s}^{2}\end{array}$ \\
\hline & & & & A \\
\hline & & & 0.02 eye (2) positive stiff system & $\begin{array}{c}\mathrm{m} / \mathrm{s} \\
\mathrm{m} / \mathrm{s}^{2}\end{array}$ \\
\hline & \multirow{3}{*}{ Measurement noise $(R)$} & \multirow{3}{*}{$1.1 I_{5 \times 1}$} & 1.1 eye (3) negative stiff system & $\mathrm{m} / \mathrm{s}$ \\
\hline & & & & A.s \\
\hline & & & 1.1 eye (2) positive stiff system & $\begin{array}{c}\mathrm{m} \\
\mathrm{m} / \mathrm{s}\end{array}$ \\
\hline \multirow{3}{*}{ System } & \multirow{2}{*}{ Mass $m$} & & 30 (isolation table) & \multirow{2}{*}{$\mathrm{kg}$} \\
\hline & & & 52 (middle table) & \\
\hline & Actuator coefficient $\left(k_{i}\right)$ & & 45 (both tables) & N/A \\
\hline
\end{tabular}

of measurement were initialized to state the process of prediction in numerical simulation.

$$
\begin{aligned}
X & =\left[\begin{array}{c}
x_{1} \\
\dot{x}_{1} \\
x_{2} \\
\dot{x}_{2} \\
{\left[\widehat{i}_{2} d t\right.}
\end{array}\right], A=\left[\begin{array}{ccccc}
0 & 1 & 0 & 0 & 0 \\
\frac{k_{2}}{m_{1}} P_{p} & 0 & -\frac{k_{2}}{m_{1}} P_{p} & 0 & 0 \\
0 & 0 & 0 & 1 & 0 \\
-\frac{k_{2}}{m_{2}} P_{p} & 0 & \frac{k_{2}}{m_{2}} P_{p} & 0 & 0 \\
0 & 0 & 0 & 0 & 0
\end{array}\right], \\
B & =\left[\begin{array}{cc}
0 & 0 \\
\frac{k_{1}}{m_{1}} & -\frac{k_{2}}{m_{1}} \\
0 & 0 \\
0 & \frac{k_{2}}{m_{2}} \\
0 & 1
\end{array}\right], u=\left[\begin{array}{l}
i_{1} \\
\hat{i}_{2}
\end{array}\right], C=\left[\begin{array}{lllll}
0 & 0 & 1 & 10
\end{array}\right],
\end{aligned}
$$

where the symbols denote exactly the same meanings as presented in Section 3.

Table 1 shows the experimental parameters and the Kalman filter $(\mathrm{KF})$ values used in the experiments and simulations. The absolute values of these parameters were kept exactly the same during the experiments and simulation.

In the numerical simulation, the developed dynamic system is considered applying to white Gaussian noise, Kalman filter estimated distances, and displacement of the system as the feedback variables. The simulated results of velocity as well as the position of the setup are taken at the steadystate condition. These results of velocity and position show that the estimated values through the estimation of the Kalman filter were better with a lower level of noisy signal when compared to a single direct measurement (Figure 5). It is also found that the feedback control system based on the calculated displacement of the Kalman filter shows a system with lower-level deviation than the actual measured signal.

The simulated results of the influences of the Kalman filter $(\mathrm{KF})$ on a control system are depicted in Figure 6. On the other hand, Figure 5 presents the simulated position of a proportional-derivative (PD) controlled system at steady state, where the displacement is considered to be a feedback variable. It is found that the Kalman filter can be used to estimate velocity and displacement signals with lower levels of noise than the measured signal. It is seen that the feedback control depending on the measured signal gives a system with a higher dynamic deviation. In contrast, if the feedback mechanism of a control system depends on the estimation of the Kalman filter, then the control system deviations are decreased significantly (Figures 5 and 6).

\section{Experimental Methodology}

4.1. Developed Experimental Setup. In this work, the developed system of isolation for vibration consists of a table for vibration isolation and a table for mass placed at the middle of the experimental setup (Figure 7). These two tables are vertically attached using bearings at the four corners of each table.

In this experimental setup, the tables are free to move along the axes of horizontal (motion of translational) and vertical to make three degrees of freedom system ( 2 horizontal and 1 vertical axis direction motions). The single axis ( $x$ -axis) motion is controlled, and the motions of the other axes are assumed to have no effect on the controlled motion. The contact areas between the tables and supports are grease lubricated so that the frictional effects in the control phenomena become negligible. The middle mass motion is controlled by voice coil motor that was set up between the middle mass and the floor, while the voice coil motor that controls the table for vibration isolation with respect to the middle table was set between the two tables. Two MEMS accelerometers are separately attached to the two tables for 

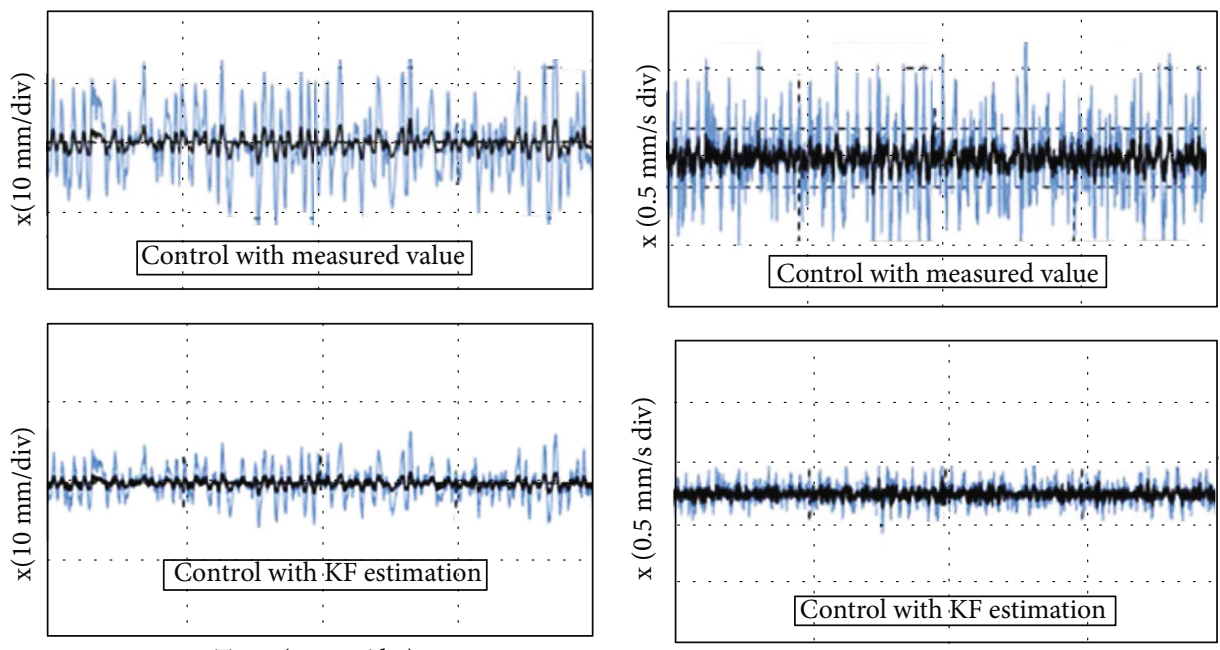

Time $(0.5 \mathrm{sec} / \mathrm{div})$

Measured

Time $(0.5 \mathrm{sec} / \mathrm{div})$

- Estimated

(a)

(b)

Figure 5: Effect of the Kalman filter on the accuracy of the signal subjected to white Gaussian noise (simulated): (a) signal for displacement and (b) signal for velocity.
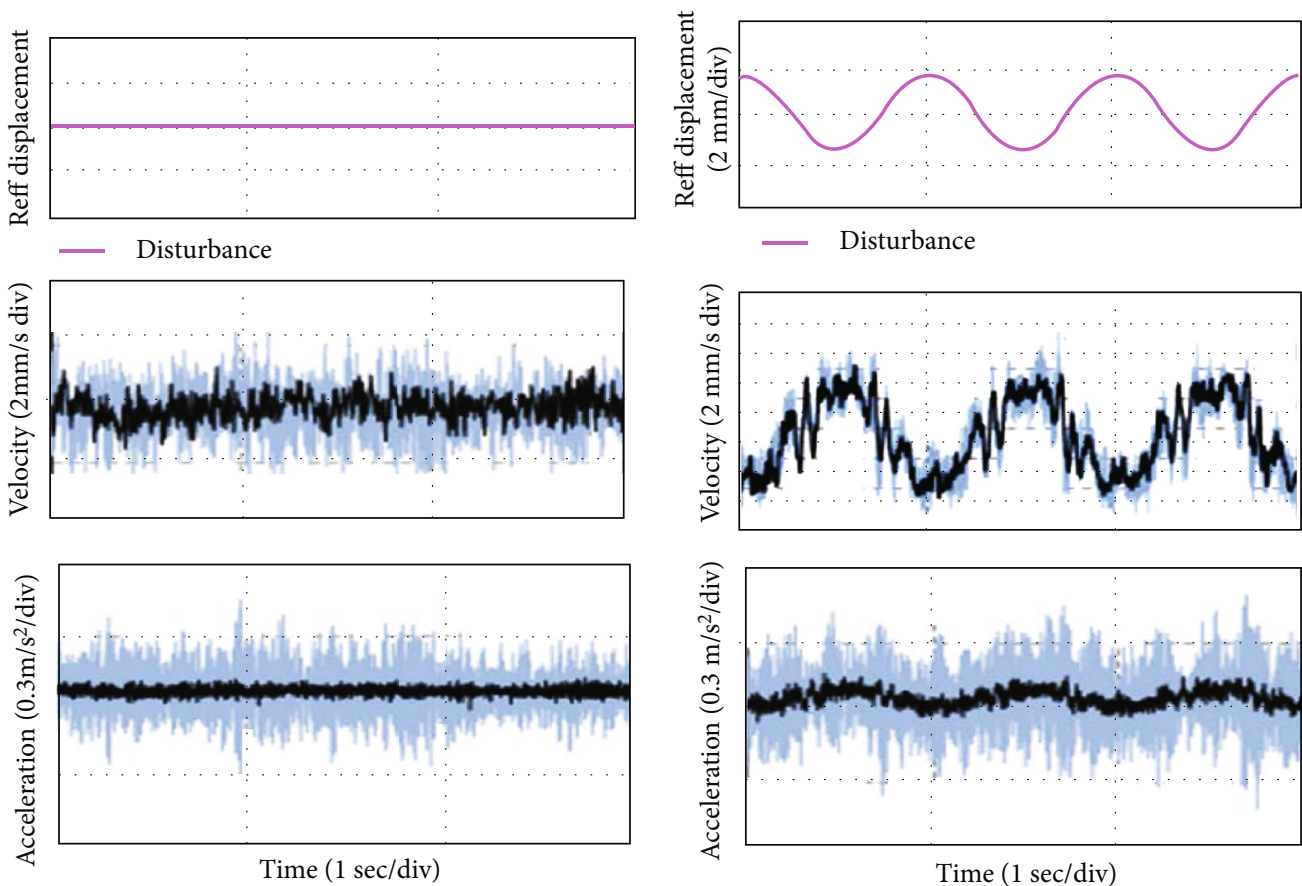

- Measured

— Measured

- Estimation

(a)

(b)

Figure 6: Measured value and estimation of the Kalman filter value regarding the reference displacement of the MEMS accelerometer: (a) reference of zero and (b) reference of sinusoids.

measuring acceleration. The control algorithms are developed using a digital signal processor $\left(\mathrm{dSPACE}^{T M}\right)$.

The size of the isolation table was $620 \mathrm{~mm} \times 620 \mathrm{~mm} \times$ $10 \mathrm{~mm}$ with a mass of around $20.5 \mathrm{~kg}$. These table dimen- sions were chosen on the basis of a traditional seat for a driver in a loaded automobile. Moreover, the size of the middle table was chosen as the dimension of $530 \times 530 \times 10 \mathrm{~mm}$, and the mass was approximately $15.5 \mathrm{~kg}$. 


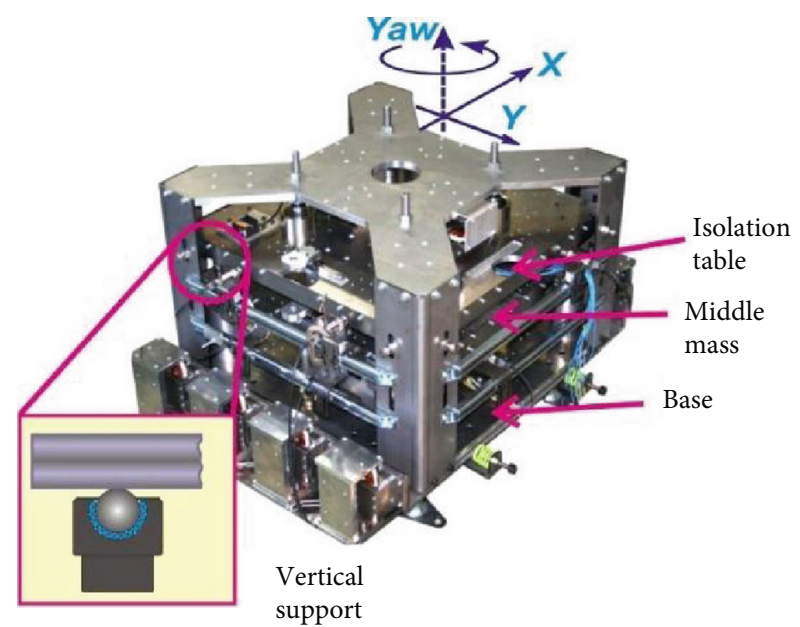

(a)

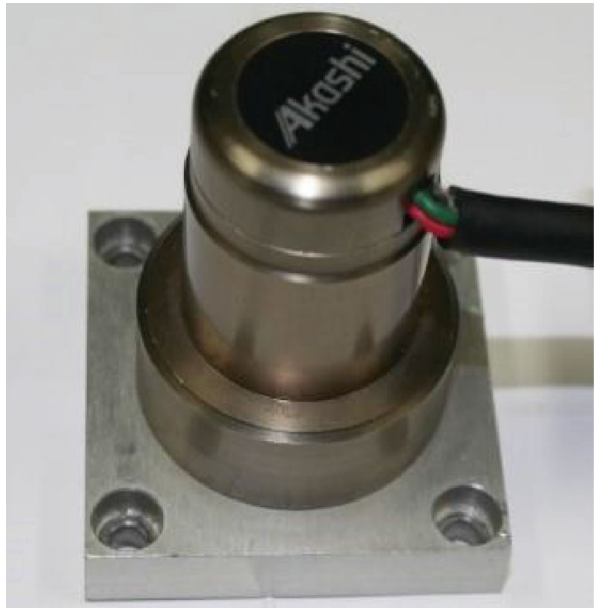

(b)

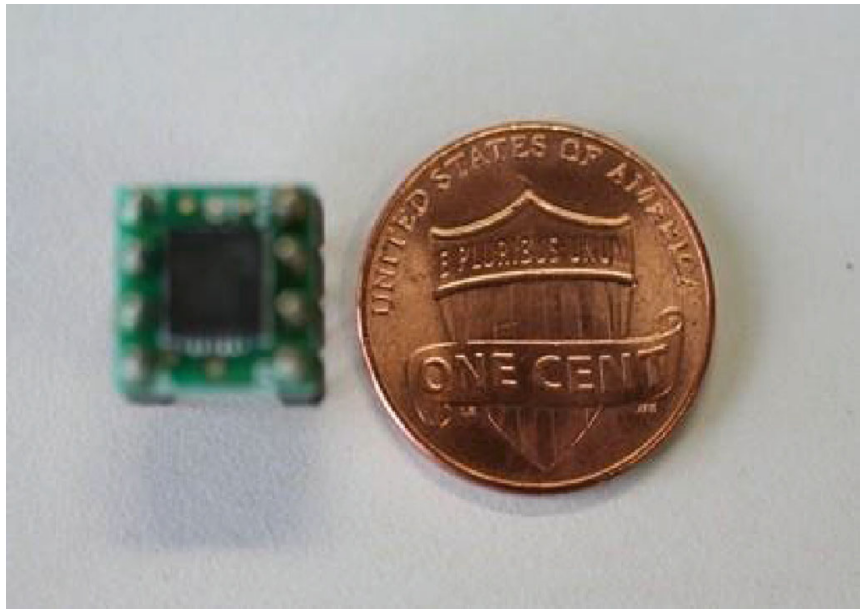

(c)

Figure 7: Experimental setup with sensors. (a) Basic experimental setup, (b) conventional servo accelerometer, and (c) MEMS accelerometer.

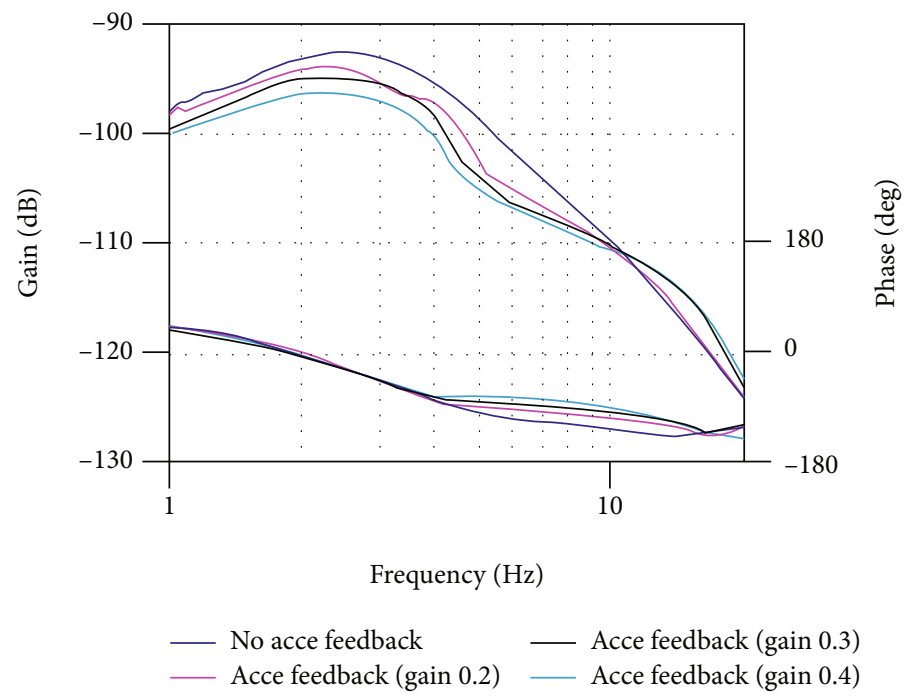

FIGURE 8: Effect of the feedback of acceleration for different gains on the responses of frequency to direct disturbance. 


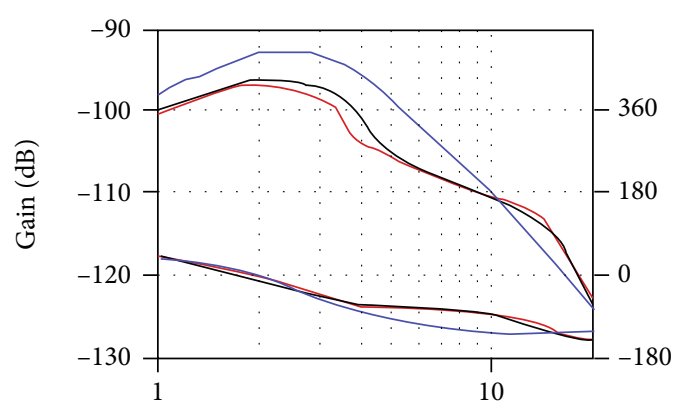

Frequency $(\mathrm{Hz})$

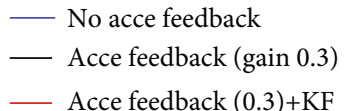

Figure 9: Effect of the feedback of acceleration based on the Kalman filter estimation for direct disturbance.

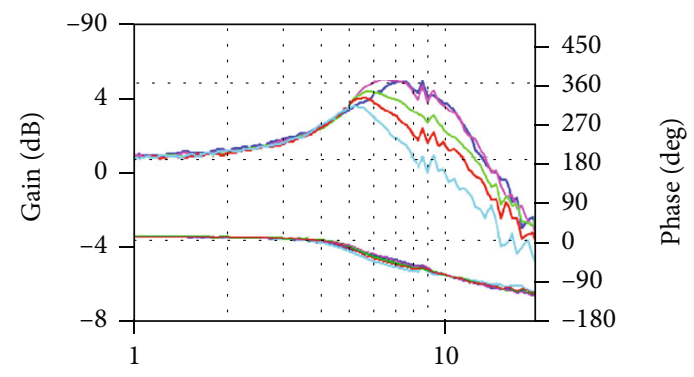

Frequency $(\mathrm{Hz})$

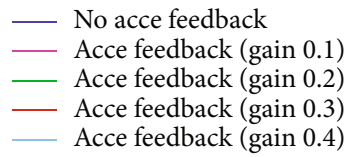

FIGURE 10: Effect of acceleration feedback of different gains on the responses of frequency to vibration of the isolation table (ground vibration).

\section{Experimental Results of Vibration Isolation System}

In this work, by using with and without acceleration feedback, several dynamic characteristics of the control system for vibration were measured. These measurements were taken for both ground vibration and direct disturbance.

Figure 8 shows the dynamic responses of the isolation system for vibration with and without the use of the feedback of the acceleration where the responses of frequency to the direct disturbance of the table for vibration isolation are presented with respect to the generated displacement as the output. In the theoretical analysis (Section 3), it was determined that a larger acceleration feedback gain $\left(P_{a}\right)$ caused a better response in the vibration isolation characteristics compared to a lower acceleration feedback gain. This theoretical finding was confirmed by the experimental

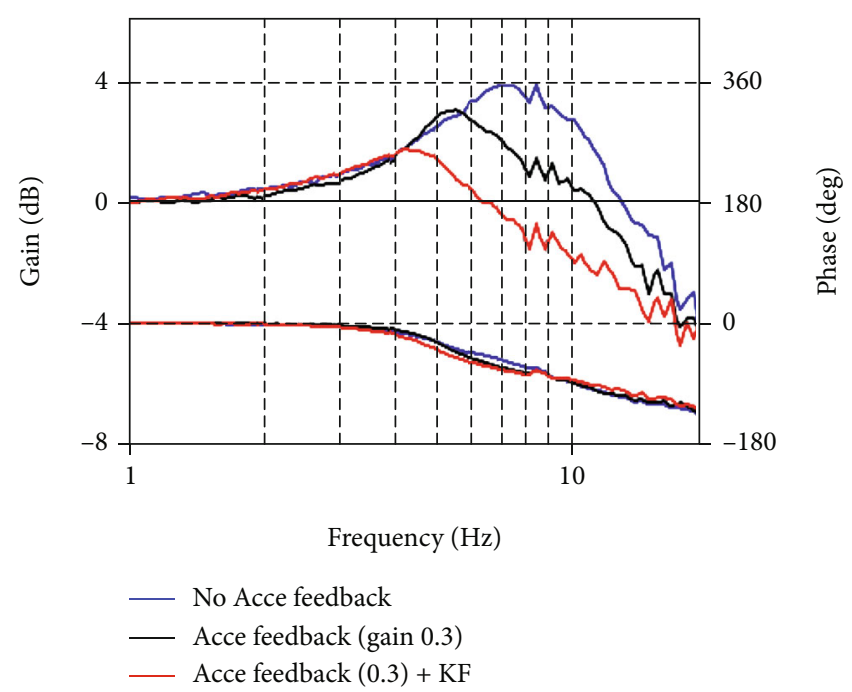

Figure 11: Effect of the feedback of acceleration based on the estimation of Kalman filter for ground vibration.

results, which are plotted in Figure 8, where the frequency responses of the vibration isolation table were measured for different $P_{a}$ values varying from 0.2 to $0.4 \mathrm{As}^{2} / \mathrm{m}$.

The peak of dynamic resonance (red line) was decreased by $6 \%$ with the estimation of Kalman filter feedback of the acceleration than the system without the use of Kalman filter (Figure 9).

The algorithm of the Kalman filter (KF) generated an estimated output that is dependent on the moving average of the immediate previously obtained outputs. Hence, in the Kalman filter estimated signals, there lie two extremely different magnitude signals and they may also be noisy. This may be one of the significant reasons for better output in the characteristics of vibration isolation through the use of the Kalman filter (KF).

In this work, the responses of frequency of the table of vibration isolation to the vibration generated by ground were calculated with and without the use of the feedback of acceleration where the gains and the phase angle were determined based on the frequency of disturbance (Figure 10). In this work, the effects of different gains of acceleration feedback on the transmissibility of ground vibration were determined.

The outcome of this work shows that ground vibration that possessed transmissibility above the resonance frequency reduced as the gain of the feedback of acceleration increased; however, it is realistic that the controlled system was stable for a certain range of $P_{a}$ values. In this work, the values of $P_{a}$ were varied from 0.1 to $0.4 \mathrm{As}^{2} / \mathrm{m}$ (Figure 10). The ground vibration transmissibility was further suppressed when the gain of the feedback of acceleration was dependent on the estimation of the Kalman filter instead of the direct measurement of signal (Figure 11). It is noted that the acceleration feedback using the Kalman filter estimation has a significant influence on the transmissibility of ground vibration. The transient peak (red-colored line, Figure 11) of the transmissibility of ground vibration with the feedback of acceleration based on the estimation 
of the Kalman filter was reduced by almost $40 \%$ compared to that of normal condition (blue-colored line, Figure 11).

\section{Conclusions}

A negative stiffness mechanism has been used for vibration isolation and realized with the developed horizontal system. The controllers for the mechanism of negative stiffness have been designed and modeled with additional acceleration feedback. In this work, MEMS accelerometers have been utilized to calculate the acceleration of the tables of the designed and modeled dynamic system. The measured noisy acceleration signals were improved by using the estimation of the Kalman filter through the MEMS accelerometer. The simulation results depicted that the estimation of the Kalman filter can estimate the values more accurately with significantly lower levels of noise when compared to the directly measured signals. The isolation characteristics of vibration of the developed dynamic system against the unpredicted disturbances were improved with acceleration feedback, which was further improved with acceleration feedback based on the estimated values of the Kalman filter. Hence, the designed and fabricated system for the isolation of vibration with the proposed techniques can be used in noisy environments where disturbances are unpredictable.

\section{Data Availability}

All the data is available to the manuscript.

\section{Conflicts of Interest}

The authors declare that they have no conflicts of interest.

\section{References}

[1] E. I. Rivin, "Vibration isolation of precision equipment," Precision Engineering, vol. 17, no. 1, pp. 41-56, 1995.

[2] M. Mourshed, F. Rashid, A. Sarker, M. Hoque, and M. H. Masud, "Single degree motion control using magnetic levitation system," in International conference on mechanical engineering and applied science, MIST, pp. 1-4, Bangladesh, February 2017.

[3] M. A. Hossain, M. A. Sufian, F. Rashid, and M. M. Z. Shahadat, "Design and fabrication of a magnetic bearing using magnetic edge effect," Engineering Research Express, vol. 3, no. 1, p. $015027,2021$.

[4] M. Yasuda and M. Ikeda, "Double-active control of microvibration isolation systems to improved performances. Application of two-dgree-of-freedom control," Transactions of the Japan Society of Mechanical Engineers C, vol. 59, no. 562, pp. 1694-1701, 1993.

[5] E. I. Rivin, Passive vibration isolation, Amer Society of Mechanical, 2003.

[6] M. E. Hoque, M. Mourshed, F. Rashid, A. Sarker, and R. Alam, "Single degree of motion control of a magnetically suspended object using analog controller," DUET Journal, vol. 3, no. 1, pp. 51-55, 2017.

[7] D. L. Trumper and T. Sato, "A vibration isolation platform," Mechatronics, vol. 12, no. 2, pp. 281-294, 2002.
[8] M. E. Hoque, F. Rashid, A. M. Saad, and M. Islam, "A vibration control system using negative stiffness mechanism," in International conference on mechanical engineering, BUET, pp. 1-7, Bangladesh, December 2015.

[9] M. G. Kibria, F. Rashid, and M. Shahadat, “Design, fabrication and performance analysis of a voice coil motor using different input signal," in IEEE International Conference on Signal Processing, Information, Communication \& Systems (SPICSCON), pp. 118-121, Dhaka, Bangladesh, November 2019.

[10] Z.-C. Qiu, C. Li, and X.-M. Zhang, "Experimental study on active vibration control for a kind of two-link flexible manipulator," Mechanical Systems and Signal Processing, vol. 118, pp. 623-644, 2019.

[11] S. Zhang, W. He, and D. Huang, "Active vibration control for a flexible string system with input backlash," IET Control Theory and Applications, vol. 10, no. 7, pp. 800-805, 2016.

[12] O. Abdeljaber, O. Avci, and D. J. Inman, “Active vibration control of flexible cantilever plates using piezoelectric materials and artificial neural networks," Journal of Sound and Vibration, vol. 363, pp. 33-53, 2016.

[13] Z. Gong, L. Ding, H. Yue et al., "System integration and control design of a maglev platform for space vibration isolation," Journal of Vibration and Control, vol. 25, no. 11, pp. 17201736, 2019.

[14] T. Mizuno, M. Takasaki, D. Kishita, and K. Hirakawa, "Vibration isolation system combining zero-power magnetic suspension with springs," Control Engineering Practice, vol. 15, no. 2, pp. 187-196, 2007.

[15] B. Yan, M. Brennan, S. Elliott, and N. Ferguson, “Active vibration isolation of a system with a distributed parameter isolator using absolute velocity feedback control," Journal of Sound and Vibration, vol. 329, no. 10, pp. 1601-1614, 2010.

[16] K. Watanabe, S. Hara, Y. Kanemitsu et al., "Combination of H/ sup/spl infin//and PI control for an electromagnetically levitated vibration isolation system," in Proceedings of 35th IEEE Conference on Decision and Control, pp. 1223-1228, Kobe, Japan, 1996, IEEE.

[17] M. Beijen, J. van Dijk, W. Hakvoort, and M. Heertjes, "Selftuning feedforward control for active vibration isolation of precision machines," IFAC Proceedings Volumes, vol. 47, no. 3, pp. 5611-5616, 2014.

[18] S. Daley, J. Hätönen, and D. Owens, "Active vibration isolation in a "smart spring" mount ${ }^{1}$ using a repetitive control approach," Control Engineering Practice, vol. 14, no. 9, pp. 991-997, 2006.

[19] T. Mizuno, H. Sekiya, Y. Ishino, and M. Takasaki, "Realization of acceleration feedback by using an active dynamic vibration absorber as a sensor in a low-frequency region," in 2015 European Control Conference (ECC), pp. 1681-1685, Linz, Austria, 2015, IEEE.

[20] T. Mizuno, T. Toumiya, and M. Takasaki, "Vibration isolation system using negative stiffness," JSME International Journal. Series C, Mechanical Systems, Machine Elements and Manufacturing, vol. 46, no. 3, pp. 807-812, 2003.

[21] M. E. Hoque, T. Mizuno, M. Takasaki, and Y. Ishino, "Application of feedforward control to a vibration isolation system using negative stiffness suspension," Journal of System Design and Dynamics, vol. 5, no. 5, pp. 777-788, 2011.

[22] M. Hossain, M. E. Hoque, F. Rashid, T. Mizuno, and Y. Ishino, "Development of a cost effective magnetic levitation system using PIC12F683 microcontroller and photo sensor," 
International Journal of Scientific Research and Engineering Development, vol. 3, no. 1, pp. 351-358, 2020.

[23] F. Moschas and S. Stiros, "Experimental evaluation of the performance of arrays of MEMS accelerometers," Mechanical Systems and Signal Processing, vol. 116, pp. 933-942, 2019.

[24] F. Mohd-Yasin, C. E. Korman, and D. J. Nagel, "Measurement of noise characteristics of MEMS accelerometers," Solid-State Electronics, vol. 47, no. 2, pp. 357-360, 2003.

[25] C. Xia, Y. Cai, Y. Ren, C. Yu, and S. Shan, "Adaptive notch filter based on Lorentz force magnetic bearing for the suppression of imbalance vibration," in Journal of Physics: Conference Series, IOP Publishing, 2019.

[26] W. Sun, H. Pan, and H. Gao, "Filter-based adaptive vibration control for active vehicle suspensions with electrohydraulic actuators," IEEE Transactions on Vehicular Technology, vol. 65, no. 6, pp. 4619-4626, 2016.

[27] T. Schuhmann, W. Hofmann, and R. Werner, "Improving operational performance of active magnetic bearings using Kalman filter and state feedback control," IEEE Transactions on Industrial Electronics, vol. 59, no. 2, pp. 821-829, 2012.

[28] H. Benzerrouk and A. Nebylov, "Aircraft's approach integrated navigation system INS/GNSS based on joint application of linear and nonlinear filtering," IFAC Proceedings Volumes, vol. 44, no. 1, pp. 1866-1871, 2011. 